\title{
ABORTO Y SALUD MENTAL DE LA MUJER
}

\author{
Justo Aznar ${ }^{1}$, German Cerdá ${ }^{2}$
}

Resumen: La existencia o no de alteraciones de la salud mental de la mujer como consecuencia del aborto es algo que suscita en el momento actual un vivo debate, pues, junto a convencidos profesionales que defienden su existencia, otros se manifiestan totalmente opuestos a ello. Para abordar este tema hemos evaluado algunas de las más recientes revisiones que nos han parecido de calidad metodológica contrastada, así como también algunos de los últimos artículos publicados. Podemos concluir que no existe un síndrome posaborto como tal, pero sí trastornos psicológicos secundarios al aborto. Finalmente, reflexionamos sobre en qué medida el sentimiento de culpabilidad que la mujer pueda experimentar por haber abortado podría influir en la presentación o no de trastornos psicológicos tras el acto abortivo.

Palabras clave: aborto, aborto y salud mental de la mujer, síndrome posaborto, trastornos psicológicos posaborto, aborto y sentimiento de culpabilidad

\section{Abortion and the mental health of women}

Abstract: The existence or the lack of alterations of mental health of women as consequence of abortion currently arises a lively debate, since, while there are convinced professionals defending its existence, others are completely opposed to it. In order to examine this issue we have evaluated some of most recent reviews considering they have contrasted methodological value, as well as some of the latest published articles. We can conclude that there is no post abortion syndrome as such, but there are secondary psychological disorders after abortion. Finally, we reflect about in which extent the feeling of guilt that woman may experiment for having aborted may influence the presentation or not of psychological disorders after the abortion act.

Key words: abortion, abortion and mental health of women, post abortion syndrome, post abortion psychological disorders, abortion and feeling of guilt

\section{Aborto e saúde mental da mulher}

Resumo: A existência ou não de alterações da saúde mental da mulher como consequência do aborto é algo que suscita no momento atual um vivo debate, pois, junto a convencidos profissionais que defendem a sua existência, outros se manifestam totalmente opostos a isso. Para abordar este tema avaliamos algumas das mais recentes revisóes que nos pareceram de qualidade metodológica comprovada, assim como também alguns dos últimos artigos publicados. Podemos concluir que não existe uma síndrome pós-aborto como tal, mas sim transtornos psicológicos secundários ao aborto. Finalmente, refletimos sobre em que medida o sentimento de culpabilidade que a mulher pode experimentar por haver abortado poderia influir na apresentação ou não de transtornos psicológicos após o ato abortivo.

Palavras-chave: aborto, aborto e saúde mental da mulher, síndrome pós-aborto, transtornos psicológicos pós-aborto, aborto e sentimento de culpabilidade

\footnotetext{
${ }^{1}$ Doctor en Medicina. Director del Instituto de Ciencias de la Vida, Universidad Católica de Valencia, Espańa Correspondencia: justo.aznar@ucv.es

${ }^{2}$ Facultad de Medicina, Universidad Católica de Valencia, Espańa
} 


\section{Introducción}

No cabe duda de que la existencia o no de alteraciones de la salud mental de la mujer secundarias al aborto es un tema que suscita en el momento actual un amplio debate médico, sociológico e incluso antropológico. En relación con ello, se constata que hay un numeroso grupo de autores que afirman la existencia de tales alteraciones(1-5), mientras que otros opinan lo contrario (6-10). ¿Cuáles pueden ser las razones para que se den resultados tan dispares?

En primer lugar, las dificultades metodológicas de estos estudios, especialmente por la existencia de factores de confusión, no siempre fáciles de controlar adecuadamente(11), como pueden ser la heterogeneidad de los grupos de mujeres evaluadas, en cuanto se refiere a edad, raza, condición social y creencias religiosas; la presencia o no, previamente al aborto, de alteraciones psicológicas; que el embarazo sea o no deseado; que cuenten o no con el apoyo de su pareja o de su familia, etc. Consecuentemente, la gran variedad de estos factores de confusión puede influir decisivamente en la heterogeneidad de los resultados obtenidos.

Pero, además, existen razones técnicas, como que gran parte de los estudios realizados son retrospectivos, ya que los estudios prospectivos, por razones éticas obvias, son difíciles de llevar a cabo.

A estas dificultades hay que añadir otras de fondo, entre ellas, que los especialistas que defienden con énfasis la existencia de un sistema posaborto, lo hagan, en muchos casos, utilizando datos de las pacientes que llegan a sus consultas reclamando atención psiquiátrica tras haber abortado y no de aquellas otras que no solamente no sufren estas alteraciones psicológicas, sino que incluso manifiestan un elevado grado de bienestar y alivio tras los abortos.

También puede influir en esta disparidad de resultados los posibles sesgos dependientes de criterios morales que tengan las personas o instituciones que realizan dichos trabajos, pues, sin duda, es muy difícil abordar un tema como este, con una carga ética tan manifiesta, sin dejarse influir por dicha circunstancia.
Por ello, al plantear nuestro estudio nos ha parecido más adecuado, y diríamos que es el único camino, evaluar algunas de las más recientes revisiones sobre el tema, las que nos han parecido de mayor calidad, para tratar de evidenciar si el aborto puede ser causa o no de alteraciones psicológicas en las mujeres que lo han sufrido y, sobre todo, constatar la posible existencia de un síndrome posaborto.

\section{Resultados}

Con esta finalidad se han evaluado cuatro revisiones(12-15) y algunos de los últimos trabajos sobre este tema.

1. La primera revisión(12) es la que el Council of Representatives de la American Psychological Association (APA) encargó a la Task Force on Mental Health and Abortion, publicada en 2008. En ella se incluyen todos los artículos escritos en lengua inglesa desde 1989 que evalúan la salud mental de las mujeres que han sufrido un aborto inducido.

El objetivo principal de esta revisión era "recoger, examinar y resumir la investigación científica hasta ese momento publicada en la que se relacionara el aborto con problemas de salud mental de las mujeres", fijándose especialmente en los siguientes aspectos: a) ¿causa el aborto algún daño sobre la salud mental de la mujer que ha abortado? b) ¿Cuál es la prevalencia de problemas de salud mental entre las mujeres de Estados Unidos que han abortado? c) ¿Cuál es el riesgo relativo de padecer problemas de salud mental secundarios al aborto comparado con el de aquellas mujeres que han terminado su embarazo por otro procedimiento? d) ¿'Se puede predecir las alteraciones psicológicas secundarias al aborto?

La principal conclusión de esta revisión sistemática es que el riesgo relativo de padecer trastornos de salud mental, tras un único aborto legal del primer trimestre de un embarazo no deseado y no realizado por razones terapéuticas, no es mayor que el que puede darse en las mujeres que dan a luz por vía natural tras un embarazo, asimismo no deseado, aunque, igualmente, se indica que en algunas mujeres que han abortado se detectan situaciones de tristeza, pena, soledad y también depresión o ansiedad, pero estiman que no existe 
evidencia suficiente para demostrar una asociación directa entre aborto y problemas de salud mental, si no se excluyen otros factores de confusión.

Otro aspecto de interés es que en ella se evalúa por primera vez si en las mujeres que abortan existen trastornos socio-psicológicos previos al aborto, pues, en caso de existir, podrían ser predictivos de una posible respuesta mental anómala tras el acto abortivo.

Lo que al parecer sí se confirma en esta revisión es la existencia de problemas mentales en las mujeres que han abortado varias veces, aunque se piensa que ello podría estar más bien relacionado con desequilibrios psíquicos previos, que pudieran predisponerlas a quedarse embarazadas sin desearlo y, consecuentemente, a tratar de terminar dichos embarazos con un aborto.

Se concluye que el que la mujer pueda tener problemas de salud mental previamente a abortar puede ser el principal factor de riesgo para sufrir trastornos psicológicos tras un aborto, aunque se manifiesta que son necesarios estudios más definitorios para determinar la posible relación entre aborto y salud mental de las mujeres, dada la diversidad y complejidad de sus circunstancias, por lo que estiman que en este momento sacar conclusiones en uno u otro sentido parece prematuro.

2. Paralelamente a esta versión se han publicado cinco artículos $(4,9,17-19)$ que a nuestro juicio complementan lo en ella expuesto.

En el primero, Taft y Watson(9), en Australia, incluyen en su estudio a 9.683 mujeres de entre 22 a 27 ańos, detectando la existencia de asociación entre aborto y depresión, aunque también la observan en mujeres que han tenido dos o más partos, en relación con las que no los han tenido, lo que de alguna forma resta fuerza a sus conclusiones sobre la existencia de alteraciones psicológicas posaborto.

En el segundo, Dingle y col.(16), también en Australia, tras ajustar diversos factores de confusión, comprueban que las mujeres que han tenido un aborto tienen tendencia al tabaquismo y al consumo de drogas, exceptuando la marihuana, así como tendencia a padecer depresión y ansiedad, cuando las comparan con el grupo de mujeres que no han abortado, aunque a este hallazgo se le puede restar valor si se tiene en cuenta que también muestran tendencia la tabaquismo y al consumo de drogas, excepto marihuana, las mujeres que han perdido un embarazo, cuando se comparan con el grupo de mujeres que nunca han estado embarazadas.

El tercero es el de Fergusson y col.(17) que evaluaremos en detalle más adelante.

El cuarto es el realizado por Pedersen y col.(4), en Noruega, en el que se valora la posible asociación entre aborto y depresión en dos grupos de mujeres, uno de menores de 20 años y otro de hasta 27 , incluyendo 40 mujeres que han abortado, 27 que han tenido un niño tras un parto normal y 700 que no han estado embarazadas en su adolescencia y juventud. Los autores concluyen que no existe diferencias en relación con la tendencia a la depresión entre los tres grupos. Pero, en cambio, las mujeres que habían abortado hacia la mitad de la década de los 20 años mostraban índices de depresión significativamente mayores que las mujeres que no habían estado nunca embarazadas, pero no mayores que las que habían dado a luz por vía normal en esa misma época.

El quinto artículo es el de Steinberg y Russo(10), en el que se detecta que las mujeres que habían abortado no tenían mayor incidencia de ansiedad y otros trastornos psicológicos que las que habían parido normalmente, pero, en cambio, en un análisis posterior, encuentran que las mujeres que habían abortado varias veces sí mostraban una mayor incidencia de ansiedad que quienes no lo habían hecho.

3. La segunda revisión evaluada(13) se centra especialmente en los problemas de salud mental secundarios al aborto a más largo plazo, pues se incluyen solamente estudios en los que el seguimiento de las mujeres tras el aborto ha sido de 90 días o más. En ella se evalúan 700 artículos, de los que solamente en 21 se incluye un grupo control.

Los autores concluyen que no se puede establecer de forma fehaciente la existencia de una relación entre aborto y problemas de salud mental tardíos 
en las mujeres que han abortado, especialmente si se tiene en cuenta que a mayor calidad de los estudios evaluados se detecta menor asociación entre aborto y riesgo mental de las mujeres que lo han padecido.

4. En la tercera revisión(14), Priscilla Coleman evalúa todos los artículos publicados en lengua inglesa, publicados entre 1995 y 2009, de los que selecciona para su consideración final 22 artículos, 15 de Estados Unidos y 7 de otros países, que incluyen un total de 877.181 mujeres, distribuidas en tres grupos: a) uno de mujeres sanas no embarazadas; b) otro de mujeres embarazadas sanas que han dado a luz un niño vivo normal y c) un tercero que incluye a 163.831 mujeres que han abortado.

Esta revisión sistemática pone de manifiesto que las mujeres que han abortado tienen un $81 \%$ más de probabilidades de padecer problemas mentales que las que no lo han hecho. Además, la posibilidad de sufrir problemas de ansiedad es en ellas un 34\% mayor, y un 37\% la de sufrir depresión. También es un $110 \%$ mayor la posibilidad de caer en el alcoholismo y un 220\% mayor la de consumir marihuana.

Pero, a nuestro juicio, el dato más relevante que aporta esta revisión es que el 10\% de las mujeres que padecen algún trastorno de salud mental han abortado con anterioridad a la aparición de los síntomas clínicos.

5. La cuarta revisión(15) es de la Academy of Medical Royal Colleges y del National Collaborating Centre for Mental Health, publicada en diciembre de 2011. En esta amplia revisión participan una veintena de profesionales o grupos de trabajo, centrando fundamentalmente su estudio en evaluar los trastornos de salud mental en mujeres que han sufrido un aborto legal tras un embarazo no deseado.

Las preguntas a las que esta revisión quiere responder son: a) ¿cuál es la prevalencia de problemas de salud mental en las mujeres que han abortado? b) ¿A qué puede deberse que los resultados sobre aborto y salud mental de las mujeres que han abortado sean tan poco concluyentes? c) ¿¿Son los problemas de salud mental más frecuentes en las mujeres que han abortado que en las que tienen un embarazo no deseado y han dado a luz naturalmente?

Sus principales conclusiones son: a) los problemas de salud mental de las mujeres de la población general después de un parto o después de un aborto son similares; b) los embarazos no deseados que terminan dando a luz por vía natural se asocian con un aumento de riesgo de problemas de salud mental; c) la incidencia de problemas de salud mental en las mujeres con un embarazo no deseado que han dado a luz por vía natural es del mismo orden que la de quienes han abortado; d) el factor predictivo de mayor impacto sobre la posibilidad de que se den problemas de salud mental tras un aborto es la existencia de una historia de trastorno mental previa al mismo; e) los autores parecen constatar la existencia de factores externos a las mujeres que abortan, que pueden asociarse a la mayor incidencia de problemas de salud mental secundarios al aborto, como puede ser la presión de la pareja para que la mujer aborte, la actitud negativa de la sociedad en general hacia el aborto o experiencias personales negativas de la mujer en relación con el aborto; y f) desde un punto de vista técnico, los autores sugieren que los meta análisis realizados para evaluar la posible asociación del aborto con problemas de salud mental de las mujeres son, en general, de baja calidad y con riesgo de tener sesgos objetivos.

6. Con independencia de las cuatro revisiones sistemáticas evaluadas y de los trabajos ya comentados, son muchos otros los estudios sobre la posible relación entre aborto y problemas mentales de las mujeres que han abortado, que naturalmente aquí no podemos referir, pero si queremos detenernos en dos de ellos: el de Fergusson(17), por su indudable importancia, y el de Munk-Olsen(18), por ser, en lo que nuestro conocimiento alcanza, el último importante publicado.

El primero(17) es un estudio longitudinal que incluye una cohorte de 534 mujeres de la que nacieron 1.265 niños, en Christchurch, una región urbana de Nueva Zelanda, a los que se ha seguido desde su nacimiento hasta que cumplieron 30 años. En dicho estudio se comprueba que - según se especifica en otro posterior de los mismos autores(19) - 284 mujeres tuvieron 686 
embarazos antes de los 30 años, con 153 abortos, en 117 mujeres; 138 embarazos que se perdieron naturalmente, en 95 mujeres; 66 nacidos vivos de 52 mujeres que presentaron reacciones adversas, y 329 de 197 mujeres que no mostraron problemas médicos.

Los autores llegan a las siguiente conclusiones: a) los abortos inducidos se asocian con un aumento de problemas mentales entre 1.86 y 7.08 veces superior al de las mujeres que no han abortado; b) los abortos naturales también se asocian a un modesto pero evidente aumento de problemas mentales, incremento que se podría cifrar entre 1.76 y 3.30 veces superior; c) los nacimientos ocurridos tras un embarazo no deseado o tras reacciones adversas durante el embarazo se asocian con un pequeño incremento en el riesgo de problemas mentales, excepto alcoholismo; d) la asociación entre problemas mentales tras un embarazo normal es débil e inconsistente; e) en las mujeres que han abortado, el riesgo de tener problemas de salud mental aumenta un 30\% en relación con las que no lo han hecho, y f) los trastornos de salud mental atribuibles al aborto representan entre el $1,5 \%$ y $5,5 \%$ de la totalidad de los trastornos mentales de las mujeres.

El segundo estudio es el de Munk-Olsen(18). En él se utilizan datos del registro civil danés, que incluye jóvenes y mujeres sin problemas mentales, durante el periodo de 1995 a 2007, que han sufrido un aborto del primer trimestre o el nacimiento de un niño durante este mismo espacio de tiempo, evaluando si estas mujeres han requerido una consulta psiquiátrica hasta 12 meses después del aborto. Los resultados muestran que la incidencia de consultas psiquiátricas por 1.000 mujeres-año, en las jóvenes y mujeres adultas que habían sufrido un primer aborto, fue de 14.6 antes del aborto y de 15.2 después de él. Este mismo índice entre las mujeres que habían tenido un niño tras un embarazo normal fue de 3.9 antes del embarazo y de 6.7 después del parto. Es decir, el índice de consultas psiquiátricas no difería sustancialmente antes y después de un aborto, pero sí se incrementaba significativamente respecto de las mujeres que tuvieron un parto normal.

Sobre este último trabajo se centran unos críticos comentarios de Priscilla Coleman, en los que se apunta que la incidencia de problemas psiquiátricos en la visita previa a un aborto es excesivamente elevada, alrededor de tres veces superior a la que presenta la población normal, por lo que Coleman sugiere que dicho incremento puede ser debido a que la mujer ya estaba inmersa en el estado de ansiedad que puede darse en los días previos al aborto, tras un embarazo no deseado. Por otro lado, un dato importante para ella es que los problemas de salud mental son significativamente mas altos después de un aborto $(15,2 \%)$, que después de un parto normal $(6,7 \%)$.

Además, un aspecto a nuestro juicio importante, y que no es tenido en consideración en el estudio de Munk-Olsen(18), es que la posible relación entre aborto y problemas mentales no se evalúa en mujeres que han tenido abortos repetidos que, como se sabe, son las que más problemas mentales posaborto presentan. También es una objetiva limitación de este estudio que solamente se sigue a las mujeres durante un año tras el aborto o parto normal, cuando existe evidencia de que muchos problemas psiquiátricos asociados al aborto no se manifiestan sino hasta varios años después de que la mujer haya abortado. Consecuentemente, el estudio de Munk-Olsen(18) debería ser evaluado teniendo en cuenta las limitaciones anteriormente expuestas.

\section{Discusión}

Al evaluar la posible relación entre aborto y trastornos de la salud mental de las mujeres, destaca en primer lugar el gran número de trabajos existentes sobre el tema, aunque, a nuestro juicio, muchos de ellos son de escasa calidad y muestran limitaciones metodológicas significativas.

La primera conclusión que creemos se puede obtener es que no se puede afirmar que exista un síndrome posaborto, pero sí se detecta en las mujeres que han abortado un aumento de problemas psicológicos especialmente si han padecido abortos repetidos, aunque en otros trabajos se concluye que el aborto ha significado un alivio para las mujeres que han abortado. Que existan resultados $\tan$ contradictorios puede, a nuestro juicio, deberse a la existencia de factores de confusión que no siempre se han evaluado convenientemente, entre los que cabría considerar: a) la heterogenei- 
dad de los grupos de mujeres que se comparan; b) los sesgos que las muestras puedan tener; c) la inadecuada valoración de la historia médica de las mujeres, especialmente en lo que se refiere a sus circunstancias reproductivas y a su salud mental; d) el distinto contexto en que el aborto se haya realizado, y e) incluso posibles sesgos en la interpretación de los datos, especialmente condicionados por las características ideológicas de las personas o instituciones que hayan realizado el estudio.

Por todo lo anterior, nuestra opinión es que, en el momento actual, hay que determinar de la forma más objetiva la posible relación entre aborto y problemas de salud mental de las mujeres que han abortado, especialmente promoviendo estudios en los que se controlen adecuadamente los factores de confusión, aunque para poder llevar a cabo este tipo de trabajos se tropieza con las muchas, por no decir ineludibles, dificultades éticas que ello conlleva.

Pero, adicionalmente, nos parece de interés afirmar lo siguiente. Por algunos se defiende la existencia de un síndrome posaborto(20), lo que es cuestionado por otros(21). Sin embargo, antes de proseguir, es necesario precisar un concepto médico: ¿qué se entiende por síndrome en medicina? Se define como tal el conjunto de signos y síntomas que configuran una enfermedad. Ateniéndonos a esta definición, estimamos que no existe un síndrome posaborto, pues no se puede evidenciar que después del aborto exista un conjunto de síntomas y signos que constituyan una enfermedad para la mujer que ha abortado. Lo que sí nos parece es que en muchas mujeres que han abortado existen trastornos de salud mental que constituyen para ellas una negativa secuela. Es decir, síndrome posaborto no, pero trastornos psicológicos sí.

A nuestro juicio, uno de los hechos que puede influir más decisivamente para evaluar la existencia o no de una relación entre aborto y problemas de la salud mental de la mujer que ha abortado es la percepción que ella pueda tener de lo que significa el aborto. El sentimiento de culpabilidad, como una posible causa de trastornos de salud mental de la mujer que ha abortado, ya ha sido consi- derado por algunos autores. En efecto, Fergusson y col.(22) comentan que entre las mujeres que presentaban trastornos psicológicos tras el aborto algunas mostraban un elevado sentimiento de culpabilidad cuando abortaron, lo que nos parece un dato de interés al valorar los posibles efectos secundarios negativos psicológicos posteriores al aborto. La percepción que la mujer pueda tener de que con el aborto ha terminado con la vida de un ser humano, en este caso un hijo suyo, puede ser un factor importante, si no decisivo, para desencadenar los trastornos psicológicos que pueden darse con posterioridad al mismo. Ello puede estar avalado porque no solamente no todas las mujeres presentan este tipo de alteración psicológica, sino que incluso, en algunos casos, muestran una sensación de alivio tras abortar(23). Esta contraposición de efectos secundarios, trastorno psicológico o sensación de alivio, debe estar fundamentalmente condicionada por la percepción del sentido ético que la mujer tenga de lo que ha realizado.

\section{Conclusiones}

1. Para definir una posible relación entre aborto y salud mental de las mujeres, no nos parece adecuado utilizar datos personales, derivados de la atención directa de los especialistas a mujeres que han abortado.

2. Para acercarse con objetividad a este problema, creemos que hay que utilizar exclusivamente datos contrastados de la literatura médica, pues, si así no se hace, siempre se encontrarán argumentos a favor o en contra de la existencia de trastornos psicológicos secundarios al aborto.

3. El sentimiento de culpabilidad que la mujer pueda tener por haber abortado puede ser un factor importante, si no decisivo, para que esta padezca trastornos psicológicos tras el acto abortivo.

4. En definitiva, nuestra opinión es que no se puede afirmar que exista un síndrome posaborto, pero sí que, tras el aborto, en muchas mujeres se pueden presentar objetivos trastornos psicológicos. 


\section{Referencias}

1. Reardon DC, Cougle JR. Depression and unintended pregnancy in the National Longitudinal Survey of Youth: a cohort study. British Medical Journal 2002; 324: 151-152.

2. Broen AN, Moum T, Bodker AS, et al. The course of mental health after miscarriage and induced abortion: a longitudinal, five-year follow-up study. BMC Medicine 2005; 3: 18.

3. Fergusson DM, Horwood LJ, Ridder E. Abortion in young women and subsequent mental health. The Journal of Child Psychology and Psychiatry 2006; 47: 16-24.

4. Pedersen W. Abortion and depression. A population-based longitudinal study of young women. Scandinavian Journal of Public Health 2008; 36: 424-428.

5. Pedersen W. Childbirth. Abortion and subsequent substance use in young women: a population-based longitudinal study. Addiction 2007; 102: 1971-1978.

6. Major B, Cozzarelli C, Cooper ML, et al. Psychological responses of women after first-trimester abortion. Archives of General Psychiatry 2000; 57: 777-784.

7. Quinton WJ, Major B, Richards C. Adolescents and adjustment to abortion: are minors at greater risk? Psychology, Public Policy and Law 2001; 7: 491-514.

8. Schmiege S, Russo NF. Depression and unwanted first pregnancy: longitudinal cohort study. British Medical Journal 2005; 331: 1303.

9. Taft AJ, Watson LF. Depression and termination of pregnancy (induced abortion) in a national cohort of young Australian women: the confounding effect of women's experience of violence. BMC Public Health 2008; 8: 75.

10. Steinberg JR, Russo NF. Abortion and anxiety. What's the relationship? Social Science and Medicine 2008; 67: 238-252.

11. Coleman PK, Coyle CT, Shuping M, et al. Induced abortion and anxiety, mood and substance abuse disorders: isolating the effects of abortion in the national comorbidity survey. Journal of Psychiatric Research 2009; 43: 770-776.

12. Council of Representatives of the American Psychological Association (APA). Report on the Task Force on Mental Health and Abortion. Washington, DC: American Psychological Association; 2008.

13. Charles VE, Polis CB, Sridhara SK, et al. Abortion and long-term mental health outcomes: a systematic review of the evidence. Contraception 2008; 78: 436-450.

14. Coleman PK. Abortion and mental health: quantitative synthesis and analysis of research published 1995-2009. The British Journal of Psychiatry 2011; 199: 180-186.

15. Induced abortion and mental health. A systematic review of the mental health outcomes of induced abortion, including their prevalence and associated factors. London: Academy of Medical Royal Colleges, National Collaborating Centre for Mental Health; December 2011.

16. Dingle K, Alati R, Clavarino A, et al. Pregnancy loss and psychiatric disorders in young women: An Australian birth cohort study. British Journal of Psychiatry 2008; 193: 455-460.

17. Fergusson DM, Horwood LJ, Boden JM. Abortion and mental health disorders: evidence from a 30-year longitudinal study. British Journal of Psychiatry 2008; 193: 444-451.

18. Munk-OlsenT, LaursenTM, Pedersen CB, et al. Induced first-trimester abortion and risk of mental disorder. New England Journal of Medicine 2011; 364: 332-339.

19. Ferguson DM, Horwood LJ, Boden JM. Reactions to abortion and subsequent mental health. The Britisch Journal of Psychiatry 2009; 195: 420-426.

20. Gómez Lavín C, Zapata García R. Categorización diagnóstica del síndrome post aborto. Actas Españolas de Psiquiatría 2005; 33: 267-272.

21. Stotland NL. The myth of abortion trauma syndrome. Journal of the American Medical Association 1992; $268: 2078$ 2079.

22. Fergusson DM, Horwood LJ, Boden JM. Abortion and mental health. The British Journal of Psychiatry 2009; 194: 377-378.

23. Templeton A, Grimes DA. A request for abortion. New England Journal of Medicine 2011; 365: 2198-2204.

Recibido: 16 de febrero de 2013

Aceptado: 2 de agosto de 2013 